
1

\title{
Determination of the Low-Abundant Protein Biomarker hCG from Dried Matrix Spots using Immunocapture and Nano Liquid Chromatography Mass Spectrometry
}

\author{
Cecilie Rosting, Elin Vyvy Tran, Astrid Gjelstad and Trine Grønhaug Halvorsen \\ Department of Pharmaceutical Chemistry, School of Pharmacy, University of Oslo, P.O. Box 1068 \\ Blindern, 0316 Oslo, Norway \\ e-mail: Cecilie Rosting: Cecilie.rosting@farmasi.uio.no, Elin Vyvy Tran: elinvyvytran@gmail.com, \\ Astrid Gjelstad: astrid.gjelstad@farmasi.uio.no,Trine Grønhaug Halvorsen: trinegha@farmasi.uio.no
}

Corresponding author:

Trine Grønhaug Halvorsen

Dept. Pharmaceutical Chemistry

School of Pharmacy

P.O Box 1068 Blindern

0316 Oslo

Norway

Phone: +47 22855735

Fax: +4722854402

E-mail: trinegha@farmasi.uio.no 


\section{Abstract}

Using LC-MS/MS for determination of low-abundance protein biomarkers from dried blood spots is challenging due to the combination of low biomarker levels (low pM-level) and small sample volumes (typically less than $50 \mu \mathrm{L}$ ). In the present paper it is demonstrated that use of state-of-the-art nano liquid chromatography triple quadrupole mass spectrometry in combination with immunoaffinity sample clean-up enable determination of the low abundance biomarker human chorionic gonadotropin (hCG) from four different biological matrices (whole blood, serum, plasma and urine) at its upper reference level (low pM). Detection limits for hCG was determined for all matrices from both commercially available non-soluble DBS sampling material (DMPK-C) and the water-soluble material carboxymethyl cellulose $(\mathrm{CMC})$. The detection limits $(\mathrm{S} / \mathrm{N}=3)$ were ranging from $5.0 \mathrm{IU} / \mathrm{L}$ (14.5 pM; whole blood) to $10.5 \mathrm{IU} / \mathrm{L}$ (30.5 pM; urine) for DMPK-C and from $2.1 \mathrm{IU} / \mathrm{L}$ (6.1 pM; urine) to 6.4 IU/L (18.6 pM; plasma) for CMC. A brief evaluation was performed for both sampling materials using serum as matrix resulting in sufficient linearity $\left(r^{2} \geq 0.93\right.$, range $20-1000 \mathrm{IU} / \mathrm{mL}$ (58-2900 pM) for DMPK-C and 10-1000 IU/mL (29-2900 pM) for CMC), repeatability (RSD\%=13-31\%) and accuracy (95106\%). To demonstrate the applicability of the method to real samples, a serum sample from a patient previously diagnosed with cancer was also analyzed using both sampling materials. The concentration levels found using the two materials were similar $(5280 \pm 595 \mathrm{IU} / \mathrm{L}(15312 \pm 1726 \mathrm{pM}$, $\mathrm{n}=3))$ in the DMPK-C spot and $5060 \pm 430 \mathrm{IU} / \mathrm{L}(14674 \pm 1247 \mathrm{pM}, \mathrm{n}=3))$ in the CMC spot). All in all this demonstrated that the tools for determination of low abundance biomarkers at upper reference level from dried matrix spots now is available through a combination of immunoaffinity enrichment and state-of-the-art LC-MS/MS.

\section{Keywords}

nanoLC-MS/MS - bottom-up analysis - immunoaffinity - dried blood spots - low abundance biomarker 


\section{Introduction}

Dried blood spot (DBS) sampling has gained increased interest the latest years due to the ease of sampling and increased stability of most analytes in dried form. Even though the main advantages in DBS sampling are related to the low invasiveness of drawing blood from a finger or heel, the use of other biological matrices than whole blood is also of interest. This is mainly due to the increased storage stability and low sample and storage volume which may be beneficial both in pharmacokinetic studies using small animals and in control of substances of abuse[1-4]. The term dried matrix spots (DMS) covers sampling of all the different biological matrices.

Combining DMS with LC-MS based protein analysis has introduced an additional challenge: high detection limits (LOD) due to the limited dynamic range of the LC-MS and the low DMS sampling volume[5, 6]. Most work has therefore been done on proteins with reference level (level seen in healthy individuals) in the $\mathrm{nM}$-range $(50-600 \mathrm{nM})[7,8]$. Methods, which provide low LODs of proteins from micro volumes of biological samples, are therefore needed in order to enable detection of for instance low-abundance protein markers.

By applying state-the-art LC-MS technology combined with selective sample clean-up (peptide SISCAPA (Stable Isotope Standards and Capture by Anti-Peptide Antibodies)) and nanoscale LCMS/MS Jung et al. recently demonstrated that ATP7B protein could be quantified low pM-levels from DBS using twenty $3 \mathrm{~mm}$ punches (approx. $3.7 \mu \mathrm{L}$ per disc) of each spot per analysis[9]. It would therefore be of great interest to evaluate if immunoaffinity clean-up of whole protein together with nanoLC-MS/MS could provide similar results for other sample matrices and other low abundance protein markers using only $15 \mu \mathrm{L}$ of blood spots.

The possibility of matching the reference levels of low abundance biomarkers from DMS could be demonstrated using any available protein. Human chorionic gonadotropin (hCG) is a low abundance biomarker with upper reference level in the low pM-range; 3.2-21.5 pM (depending on age, gender and biological matrix)[10]. hCG-levels is elevated during pregnancy and is used for establishing 
pregnancy. hCG-levels are also elevated in certain cancers (e.g. ovarian and testicular cancer), and is useful to monitor during abnormal pregnancies $[10,11]$. In addition, hCG is on WADAs prohibited list for male athletes and by such interesting to analyze in a doping perspective. Our group has extensive knowledge on analysis of hCG using the bottom-up principle [12-18]. Due to all this, hCG was chosen as model biomarker in the present work.

Two papers previously published by our group were used as starting point $[13,18]$. The first paper describes method development for determination of hCG and its variant from serum using LC-SRMMS, and the second paper introduces water-soluble material for DBS in protein analysis using hCG as a model protein. Magnetic bead-based immunoaffinity sample clean-up and microbore LC-SRM-MS separation and detection were used in both works. hCG could be determined at upper reference level from $1 \mathrm{~mL}$ serum and urine (LOD $5 \mathrm{IU} / \mathrm{L}(14.5 \mathrm{pM})$ and $2 \mathrm{IU} / \mathrm{L}(5.8 \mathrm{pM})$, respectively) [13] while a rather high LOD was achieved (100 IU/L (290 pM)) in the DBS work[18].

In the present paper it was investigated if detection of low abundance biomarkers at reference level was possible from only minute volumes of biological samples using state-of-the-art LC-MS equipment (i.e. nanoLC-MS/MS) combined with the aforementioned selective sample preparation based on immunoaffinity. Four different biological matrices: whole blood, serum, plasma and urine were evaluated. This would make a valuable contribution to the use of DMS in protein analysis since previously to our knowledge only whole blood has been applied as matrix for targeted protein analysis. LOD based on a signal-to-noise ratio of three was determined for all four matrices after spotting $15 \mu \mathrm{L}$ of sample on two different sampling materials: commercially available cellulose based material (DMPK-C) and water-soluble carboxymethyl cellulose (CMC) material. In addition, a brief evaluation of the method performance (linearity, and with-in day repeatability and accuracy) was performed using serum as spotting matrix. Finally, determination of hCG in a serum sample from a person diagnosed with testicular cancer was performed to demonstrate the potential use in real applications. 


\section{Materials and methods}

\section{$2.1 \quad$ Chemicals}

Pregnyl ${ }^{\circledR}$ (hCG) 5000 IU/ampulla was obtained from Organon (Oss, The Netherlands). Tosyl phenylalanyl chloromethyl ketone (TPCK) treated trypsin $\geq 10000$ BAEE IU/mg protein, ammomium bicarbonate $(A B C) \geq 99.5 \%$, iodoacetic acid (IAA) $\geq 98 \%$, 1,4-dithiothretiol (DTT) $\geq 99.5 \%$, formic acid (FA) $98 \%$, trifluoroacetic acid (TFA) $\geq 98 \%$, poly(ethylene glycol) (PEG) $2000020 \%$ (w/v), disodium phosphate dihydrate $(98.5-101.0 \%)$, Trizma ${ }^{\circledR}$ base $\geq 99.9 \%$, Tween ${ }^{\circledR} 20$, and the internal standard AQUA peptide (amino acid sequence: VLQGVLPALPQVVCNY[R_13C6_15N4]) were all purchased from Sigma-Aldrich (St. Louis, MO, U.S.A.). The international reference standard for intact hCG (standard code 99/688) was obtained from National Institute of Biological Standards (Hertfordshire, UK). Acetonitrile (MeCN) MS-grade, sodium chloride $\geq 99.5 \%$, potassium chloride $\geq 99.5 \%$, potassium dihydrogen phosphate $\geq 99.5 \%$, and hydrochloric acid $37 \%$ were obtained from Merck (Darmstadt, Germany).

Human serum and plasma were obtained from healthy volunteers from Oslo University Hospital, Ullevaal (Oslo, Norway). Whole blood and urine were donated by one person. The whole blood was collected in BD vacutainer $\mathrm{K}_{2}$ EDTA tubes.

Anti-hCG (monoclonal antibody E27) coated on magnetic beads was donated by the Central Laboratory, Norwegian Radium Hospital, Oslo University Hospital (Oslo, Norway).

\subsection{Preparation of standards and spiked solution}

Preparation of hCG Stock Solution. A stock solution of Pregnyl-hCG was prepared by dissolving one ampulla of Pregnyl (5000 IU) in $1 \mathrm{~mL}$ of ion-exchanged water. This stock solution was dispensed into smaller volumes, stored at $-32{ }^{\circ} \mathrm{C}$ and thawed before dilution in whole blood, plasma, serum, or urine. All samples were prepared freshly on the day of experiment. The hCG sequence can be found in 
Supplementary material Figure S1 together with sequence of hCG- $\beta T 5$, the tryptic signature peptide

122 monitored.

123 Internal Standard Solution. One ampulla containing $1 \mathrm{nmol}$ peptide was added $20 \mu \mathrm{L}$ of $10 \%$ (v/v) FA 124 in water to dissolve the peptide. A volume of $180 \mu \mathrm{L}$ of $0.1 \%(\mathrm{v} / \mathrm{v}) \mathrm{FA}$ in water was then added and

125 the solution was vortexed. This solution served as a stock solution $(5 \mathrm{nmol} / \mathrm{mL})$.

126 Prior to use the cysteine residues on the AQUA peptide were reduced and alkylated in order to have

127 the same physicochemical properties as the signature peptide ( $\beta T 5)$. The reduction and alkylation 128 was performed as previously described by Lund et al. [13].

129 Preparation and analytical performance of aqueous reference samples can be found in 130 Supplementary material.

\section{3 hCG denomination}

132

In the clinical environment the common denomination for hCG is IU/L. However to be able to relate the present work to other proteins, all concentration levels are given in both IU/L and $\mathrm{pM}$. The conversion is done as described elsewhere[10] where $1 \mathrm{IU}$ (if intact hCG) is described to equal 2.9 pmol.

\subsection{Preparation of dried matrix spots (DMS)}

Fifteen $\mu \mathrm{L}$ of whole blood, plasma, serum or urine added hCG (spike volume $<1 \%$ ) was deposited on FTA $^{\circledR}$ DMPK-C cards (Whatman, Kent, UK) or on a piece of Aquacel ${ }^{\mathrm{TM}}$ hydrofiber ${ }^{\circledR}$ (water-soluble material, CMC) from Convatec (Skillman, NJ, USA). The samples were then dried for at least two hours in room temperature before punching out the entire spot. The whole spot was exerted in

141 order to circumvent potential hematocrit effect from the whole blood sample. The spots were

142 subsequently transferred to a $1.5 \mathrm{~mL}$ LoBind protein Eppendorf tube (Eppendorf, Hamburg,

143 Germany) and added $1 \mathrm{~mL}$ of phosphate buffer saline $\mathrm{pH} 7.4$ (PBS). The DMPK-C spots were 
extracted for 1 hour prior to immunoaffinity extraction while the CMC spots were dissolved using a Thermomixer at $1400 \mathrm{rpm}$ prior to immunoaffinity extraction. PBS was used for extraction/dissolution of the spots due to compatibility with the subsequent immunoaffinity extraction procedure as demonstrated in [18].

\subsection{Immunoaffinity extraction, tryptic digestion and solid-phase extraction}

Immunoaffinity extraction was performed using magnetic beads coated with the antibody E27 as prepared $[13,19]$ and described for DBS previously [18]. The only exception was extraction of the DMPK-C cards for one hour prior to transfer of the extracted sample to a new Eppendorf vial for the $1 \mathrm{~h}$ immunoextraction.

The tryptic digestion (subsequent to reduction and alkylation) was performed after the immunoaffinity clean-up as previously described[18].

Solid-phase extraction (SPE) was performed using in-house made SPE columns as described by Lund et al. [13] to enrich the sample after immunoaffinity extraction and tryptic digestion. The same SPE procedure as previously used for enrichment of the target peptide from DBS was used[18]. The complete sample volume after tryptic digestion and addition of internal standard (approx. $110 \mu \mathrm{L}$ ) was transferred to the SPE column. To enable a higher enrichment of the peptides the residue was reconstituted in $20 \mu \mathrm{L}$ compared to 50 or $30 \mu \mathrm{L}$ in the previous papers $[13,18]$.

\subsection{Nano liquid chromatography - mass spectrometry}

The previously developed LC-SRM method was transferred to a state-of-the-art nanoLC-triple quadrupole. Some changes from the previous method were introduced when transferring the method to a new analytical system (change of column chemistry and dimensions, injection volume, LC gradient and ion source). No changes were made in the SRM transitions. 
Chromatography: The chromatographic separation was in this paper performed on a nanoLC system (UltiMate $^{\mathrm{TM}} 3000$ RSLCnano System from Thermo Scientific) using an Acclaim ${ }^{\mathrm{TM}}$ PepMap $^{\mathrm{TM}} 100 \mathrm{C} 18$ column (150 mm x $0.075 \mathrm{~mm}$ i.d., $3 \mu \mathrm{m}$ ) and an Acclaim ${ }^{\text {TM }}$ PepMap ${ }^{\text {TM }} 100$ C18 column $(5 \mathrm{~mm} \times 0.30$ $\mathrm{mm}$ i.d., $5 \mu \mathrm{m}$ ) trap column. The column temperature was $35^{\circ} \mathrm{C}$. Chromeleon software (version 6.80 SR13 Build 3818) was used to control the process. Ten microliter of sample was loaded onto the trap column using a loading mobile phase consisting of $20 \mathrm{mM}$ of FA in $\mathrm{H}_{2} \mathrm{O}$ and $\mathrm{MeCN}(98: 2 \mathrm{v} / \mathrm{v})$ and a flow of $30 \mu \mathrm{L} / \mathrm{min}$. The trap column was then washed for 3 min using the loading mobile phase prior to transferal of the analytes to the analytical column using the mobile phases. The mobile phases consisted of $A, 20 \mathrm{mM}$ of FA in $\mathrm{H}_{2} \mathrm{O}$ and $\mathrm{MeCN}(95: 5 \mathrm{v} / \mathrm{v})$, and $\mathrm{B}, 20 \mathrm{mM}$ of FA in $\mathrm{H}_{2} \mathrm{O}$ and $\mathrm{MeCN}$ (5:95 v/v). The gradient ran from $5 \%$ to $20 \%$ mobile phase B in $7.5 \mathrm{~min}$, then B was kept constant at $20 \%$ for 17.5 min before the elution strength was further increased to $95 \%$ B within $0.1 \mathrm{~min}$, kept constant for $1 \mathrm{~min}$, and then returned back to starting conditions within $0.1 \mathrm{~min}$. The flow was set to $0.3 \mu \mathrm{L} / \mathrm{min}$. Both the trap and the analytical column were reequilibrated for at least 10 column volumes each. Automatic filtration of the injected samples was performed as described elsewhere using a steel filter (Replacement screen, 1/16" from Teknolab, Norway)[20].

Mass Spectrometry: MS analysis was performed in selected reaction monitoring mode (SRM) using a triple quadrupole analyzer (TSQ Quantiva, Thermo Scientific) equipped with a nano electrospray ionization interface (Nanospray flex) and operated in the positive ion mode. Spray voltage was set to $1800 \mathrm{~V}$, sweep gas (nitrogen) pressure of two arbitrary units. The capillary temperature was set to $350{ }^{\circ} \mathrm{C}$. The SRM method was based on the method previously published by Lund et al. [13], using the hCG specific tryptic peptide $\beta$ T5 (VLQGVLPALPQVVCNYR) as surrogate peptide. The selection and confirmation of this peptide as signature peptide is described in [17] . The signature peptide $\beta T 5$ (VLQGVLPALPQVVCNYR) and the internal standard (VLQGVLPALPQVVCNY $\left[\mathrm{R}_{-}{ }^{13} \mathrm{C}_{6}{ }_{-}{ }^{15} \mathrm{~N}_{4}\right]$ ) was monitored using the transitions $964.2 \rightarrow 1036.3$ and $964.2 \rightarrow 1317.8$ for the signature peptide and $969.3 \rightarrow 1046.3$ and $969.3 \rightarrow 1327.8$ for the internal standard. The collision energy was $30 \mathrm{~V}$ for all 
transitions. The collision gas (argon) pressure in Q2 was 1.5 mTorr, the Q1 and Q3 resolution both 0.7. The process was controlled by Xcalibur 3.0.63.

\subsection{Determination of detection limits}

The detection limits was determined for all four matrices using both sampling materials. The detection limits were based on a signal-to-noise $(\mathrm{S} / \mathrm{N})$ ratio of three using the average noise in the signature peptide channel at the retention time of the internal standard. Five injections of blank matrix (spiked with internal standard) and average signal intensity of five samples spiked with 20 $\mathrm{IU} / \mathrm{mL}(=58 \mathrm{pM})$ were used for calculating the concentration of hCG corresponding to $\mathrm{S} / \mathrm{N}=3$

\subsection{Method evaluation}

Method evaluation was performed for hCG in dried serum spots evaluating the following parameters: linearity using five concentration levels ( $n=3$; DMPK-C: 20, 50, 200, 500 and 1000 IU/L and CMC: 10, 50, 200, 500 and $1000 \mathrm{IU} / \mathrm{L}$ ) and 1/x weight of the calibration curve. Repeatability presented as relative standard deviation (RSD (\%)) and accuracy presented as percent of added value was performed at the lowest and highest level of the calibration curve $(n=5)$.

\subsection{Analysis of real samples}

A serum sample from a patient previously diagnosed with testicular cancer was analyzed using both sampling material $(n=3)$. First fifteen microliter of the sample was spotted on both materials, prepared as described earlier and a concentration estimate performed. As the signal intensity of this sample was approximately five times higher than the signal intensity of the highest level in the concentration curve the patient sample was diluted five times with hCG free serum before spotting fifteen microliter of diluted sample on both materials $(n=3)$. Subsequently the samples were analyzed simultaneously with the calibration curve in order to estimate the concentration level of the patient 
sample. The obtained level was multiplied by five in order to calculate the level in the undiluted

215 patient sample.

\section{Results and Discussion}

217

218

219

220

221

\subsection{Detection limits}

LODs were determined for both sampling materials and for all four biological matrices in order to evaluate the improvements in method sensitivity after introduction of the state-of-the-art nanoLCMS/MS system. Chromatograms after preparation of blank (non-spiked) DMS and DMS spiked with analyte at low levels (20 IU/L / 58 pM) are shown in Figure 1 and Figure 2 for DMPK-C and CMC, respectively. These chromatograms confirmed that the immunoaffinty sample preparation provided clean extracts, advantageous for detection of low analyte concentrations. Calculation of the LODs (set as signal-to-noise $(\mathrm{S} / \mathrm{N}$ ) ratio of 3 ) was based on the signal from these spiked samples (spiked at $20 \mathrm{IU} / \mathrm{L}$ ) and the noise at the retention time of the signature peptide from the blank samples (spiked with IS). The LODs were determined for all matrices and both sampling materials and the calculated LODs are shown in Table 1 and ranged between 2.1-10.5 IU/L (6.1-30.5 pM) depending on the biological matrix. The variation in LODs was probably due to different level of background noise obtained from the four matrices. Only small differences were observed between the two sampling materials, although CMC in general provided better LODs than DMPK-C (except for whole blood where the LOD was comparable). Overall, the signal intensity of the hCG-peptide was higher for CMC samples compared to DMPK-C samples. In addition, the noise was also higher, although for most matrices the increase in signal for hCG was higher than the increase in noise level (exception whole blood). Previously, ion enhancement is described for hCG in whole blood using CMC as sampling material [18]. If ion enhancement is present when using $\mathrm{CMC}$ and not using DMPK-C this might be the reason for the differences. Differences in matrix effects using different sampling materials have been observed using a set of model proteins in DBS w/o use of IS (submitted manuscript). These differences were however compensated for when including IS in the analysis. 
The lowest LOD was seen for urine using CMC as the sampling material, providing an LOD of $2.1 \mathrm{IU} / \mathrm{L}$ (6.1 pM). The highest LOD (10.5 IU/L (30.5pM)) was obtained for urine using DMPK-C, and the reason for the increased LOD from urine on DMPK-C was due to decreased signal of the signature peptide compared to the signal from the CMC samples. For DMPK-C, the LOD seen for urine is higher than for plasma despite the latter being a more complex matrix. However, looking into the RSD values (\%) for the spiked samples used to determine the LOD (Supplementary material Table S2), it can be seen that the RSDs (\%) from plasma were very high and hence the LOD estimates based on these values contains high uncertainty. In addition, the signal intensity of hCG in urine spotted on DMPK-C is lower compared to when spotted on CMC. Part of the reason for the high urine LOD might hence be due to differences in matrix effects using the two different sampling materials. The immunoextraction may also be more efficient from the dissolved $\mathrm{CMC}$ sample compared to when applying it to the nonsoluble DBS.

The obtained LODs were despite some variation between the matrices, all in all comparable to the detection limits seen for $1 \mathrm{~mL}$ serum and urine using the previously described analytical system [13] even though a 67 times lower sampling volume was used. The LODs also was improved $>20.4$ times compared to the previous work describing detection of hCG from $15 \mu \mathrm{L}$ DBS [18]. In general the RSDs of the area ratios used for the determination of LOD was satisfactory $(\leq 25.5 \%$ RSD, $n=5$, Supplementary material TableS2), with the exception of the plasma samples where the observed RSDs were $152 \%$ and $49 \%$ for DMPK-C and CMC, respectively. The reasons for this is not known, but it might be due to the anticoagulant used for the plasma samples, citrate phosphate dextrose-adenine (CPDA-1) was used for plasma vs. $K_{2}$ EDTA was used for whole blood. We have previously seen that the choice of anticoagulant ( $\mathrm{K}_{2}$ EDTA versus lithium heparin) affected the repeatability and matrix effects of hCG from dried blood spots using CMC as sampling media [18]. It 
signature peptide as well. Hence, if plasma spots are used it is important to evaluate the choice of anticoagulant during method development.

Nevertheless, it can be seen that by minor method refinements in combination with state-of-the-art nanoLC-MS/MS system enable similar LODs for hCG as previously seen when using conventional LCMS/MS and a 67 times higher sample volume. Sufficient repeatability for three of the four matrices was also seen.

\subsection{Method evaluation}

The main goal of the present paper was to investigate if the use of state-of-the-art LC-MS equipment made it possible to detect low abundance biomarkers at their reference level. This was done by evaluating the LODs of hCG from the different DMS. As the present method was not intended as a ready-to-go method for use in biomarker analysis of hCG a brief evaluation (linearity, with-in day repeatability and accuracy) of the performance was performed for only one matrix, serum. This was considered acceptable in order to get an indication of the performance at these low levels of analyte using low sample volumes and a miniaturized LC-MS/MS system. Serum was chosen as the matrix as a serum sample from a patient previously diagnosed with testicular cancer was available for analysis. Real samples were not available for any of the other matrices. Both sampling materials were included as previous research has shown that the water-soluble material often performs at least as good as the non-soluble cellulose material. Matrix effects have previously been evaluated qualitatively for hCG in serum and urine after immunoaffinity clean-up [13] and quantitatively for hCG in watersoluble DBS after immunoaffinity clean-up [18], showing either no matrix effects or effects that could be compensated for during quantification by an internal standard, respectively. Matrix effects were therefore not evaluated in the present work. It is worthy to note that the matrix effects were evaluated using a microbore LC-MS/MS system equipped with an C8 column opposed to the current nano system equipped with an C18 column. Some of the observed differences in LOD might hence be due to differences in matrix effects using the selected matrices and sampling materials (see above). 
However, as the repeatability at the level used for estimation of LOD (20 IU/L (58 pM)) was considered satisfactory (for all matrices except plasma; see above and in Supplementary material Table S2), possible matrix effects were considered sufficiently corrected for by the IS (except for plasma).

The stability of hCG in DMS was not evaluated in the present paper. Extensive knowledge is already available on storage of hCG, both long and short time in serum/urine $[13,21]$ and in DBS $[18,22]$.

294

295

Repeatability and accuracy was determined at the high and low level of the calibration curves (i.e the upper and lower level of quantification (LLOQ and HLOQ), n=5). Due to the slightly higher LOD of hCG using DMPK-C compared to CMC the lower level for DMPK-C was twice as high as for CMC (20 vs 10 IU/L). A representative chromatogram for lowest concentration level in the calibration curve can be seen in Supplementary material Figure S2 for both DMPK-C and CMC serum spots.

The observed RSDs (Table 2 ) were somewhat increased ( $\leq 31 \%$ ) compared to the immunoaffinity method using $1 \mathrm{~mL}$ of serum ( $\leq 19 \%)$, and the linearity was also lower $\left(r^{2} \geq 0.93\right.$ vs $\left.r^{2}>0.997\right)$. This can be explained by extra bias introduced by the dissolution or elution process of the sample from the sampling materials, although it was previously shown that the water-soluble material gave complete recovery of analyte from the dissolution step [18]. A sample volume of $15 \mu \mathrm{L}$ is also more prone to variations caused by the several preparation steps compared to a sample volume of $1 \mathrm{~mL}$ (as more analyte is available in the large sample volume). In addition, nanoLC is generally less robust than LC using microbore or conventional columns. The RSDs were also increased and the correlation coefficient lower compared to the data obtained by Jung et al. (RSD $<3 \%$ and $\mathrm{R}^{2}=0.997$, respectively) when measuring a low-abundant protein by using immunoaffinity capture and nanoLCMS/MS[9]. Jung et al. used the SISCAPA approach where the stable isotope labelled internal standard was correcting for variation caused by the immunoaffinity clean-up (post proteolysis immunocapture). In our work, the isotope labelled internal standard was added after the immunoaffinity clean-up step and was therefore not correcting for this step (pre proteolysis 
immunocapture). Improvements in the repeatability using for instance whole protein internal standard and extensive method comparison are on the other hand necessary prior to implementation in routine biomarker analysis. In addition, the use of automated sample processing equipment such as work stations/robots (96-wells format) for performing all steps from sample punching through immunoextraction, tryptic digestion and SPE will result in improvements of the method performance, as well will the development and use of more robust nano systems. Automation in the 96-wells format will also lower the costs and enable higher throughput as labor is expensive and also often rate-determining for a procedure. Using the current set-up 30 samples may be processed in parallel in less than 24 hrs (limitation: centrifuge for SPE), using two hours for dissolution/extraction and immounoextraction, overnight tryptic digestion, and additional two hours for micro SPE. The capacity of the LC-MS system is 34 samples per $24 \mathrm{hrs}$. The rate-limiting step of the current procedure is hence the sample processing but if automated in the 96-wells format the LCMS/MS analysis will be rate limiting. However, the results demonstrate that it is possible to determine low abundance protein biomarkers from dried matrix spots using only $15 \mu \mathrm{L}$ of sample with sufficient accuracy. In addition, the sample volume of DMS used in the current paper is comparable with the volume used in immunological methods but has the advantage of the increased specificity of LC-MS making it possible to perform isoform differentiation using same amount of sample as in immunological techniques.

\subsection{Analysis of a real sample}

A real sample from a patient previously diagnosed with testicular cancer was analyzed in order to demonstrate the applicability of the method on real samples. Fifteen microliter of serum was spotted on both the commercial available DBS material and the water-soluble material, and analyzed as described above $(n=3)$. As initial experiments demonstrated that the level of the patient sample was 
pM) in the DMPK-C spot and $5060 \pm 430 \mathrm{IU} / \mathrm{L}(14674 \pm 1247 \mathrm{pM})$ in the CMC spot. This demonstrates that the method can be applied to samples containing endogenous hCG, and that the two sampling materials provides similar results. The quantified levels are much higher than the reference levels in heathy humans. This demonstrate that methods used for quantification of biomarkers often need to span a broad range from the level of healthy individuals in the low pM range up to levels in the $\mathrm{nM}$ range (for hCG from $\leq 5 \mathrm{IU} / \mathrm{L}$ to $\geq 10000 \mathrm{IU} / \mathrm{L}(14.5-29000 \mathrm{pM})$ [11]. Chromatograms of the two analyses can be found in Figure 3. Inclusion of more real samples will be necessary to verify that the method can be used in a clinical setting.

\section{Conclusions}

Combining state-of-the-art nanoLC-MS/MS with dried matrix spots enables detection and quantification of the low abundance protein biomarker hCG at upper reference level (low pM) from minute volumes of various biological samples (whole blood, serum, plasma and urine). This is a considerable improvement (> 10 times) compared to previously published method for hCG in DBS and comparable to results obtained from serum using $1 \mathrm{~mL}$ of sample (both using microbore LCMS/MS system). Both commercially and non-commercially available sampling materials showed potential as sampling material for dried serum samples in quantitative analysis of hCG, but a more comprehensive method evaluation together with inclusion of more patient samples will be necessary to verify that the method can be used in a clinical setting. However, these promising results indicate that matrix spots can be a valuable sampling format for detection of low-abundant proteins despite the small sample volumes available.

\section{Acknowledgements}

Elisabeth Paus at the Norwegian Radium Hospital (Oslo University Hospital, Oslo, Norway) is greatly acknowledged for providing the patient sample, the hCG reference standard, and the antibody coated magnetic beads. 
[1] A.S. Hagan, D.R. Jones, R. Agarwal, Use of Dried Plasma Spots for the Quantification of lothalamate in Clinical Studies, Clinical Journal of the American Society of Nephrology, 8 (2013) 909914.

[2] Y. Lee, K.K.Y. Lai, S.M.H. Sadrzadeh, Simultaneous detection of 19 drugs of abuse on dried urine spot by liquid chromatography-tandem mass spectrometry, Clinical Biochemistry, 46 (2013) 11181124.

[3] W. Li, J. Doherty, S. Favara, C. Breen, J. Flarakos, F.L.S. Tse, Evaluation of plasma microsampling for dried plasma spots (DPS) in quantitative LC-MS/MS bioanalysis using ritonavir as a model compound, Journal of Chromatography B, 991 (2015) 46-52.

[4] S. O'Broin, E. Gunter, Dried-Serum Spot Assay for Folate, Clinical Chemistry, 48 (2002) 1128-1130. [5] S. Lehmann, A. Picas, L. Tiers, J. Vialaret, C. Hirtz, Clinical perspectives of dried blood spot protein quantification using mass spectrometry methods, Critical reviews in clinical laboratory sciences, 54 (2017) 173-184.

[6] V. Ignjatovic, J. Pitt, P. Monagle, J.M. Craig, The utility of dried blood spots for proteomic studies: looking forward to looking back, Proteomics. Clinical applications, 8 (2014) 896-900.

[7] A. deWilde, K. Sadilkova, M. Sadilek, V. Vasta, S.H. Hahn, Tryptic peptide analysis of ceruloplasmin in dried blood spots using liquid chromatography-tandem mass spectrometry: application to newborn screening, Clin. Chem., 54 (2008) 1961-1968.

[8] A.G. Chambers, A.J. Percy, J. Yang, A.G. Camenzind, C.H. Borchers, Multiplexed quantitation of endogenous proteins in dried blood spots by multiple reaction monitoring-mass spectrometry, Molecular \& cellular proteomics : MCP, 12 (2013) 781-791.

[9] S. Jung, J.R. Whiteaker, L. Zhao, H.-W. Yoo, A.G. Paulovich, S.H. Hahn, Quantification of ATP7B Protein in Dried Blood Spots by Peptide Immuno-SRM as a Potential Screen for Wilson's Disease, J. Proteome Res., 16 (2017) 862-871.

[10] U.-H. Stenman, A. Tiitinen, H. Alfthan, L. Valmu, The classification, functions and clinical use of different isoforms of HCG, Human Reproduction Update, 12 (2006) 769-784.

[11] U.-H. Stenman, H. Alfthan, K. Hotakainen, Human chorionic gonadotropin in cancer, Clinical Biochemistry, 37 (2004) 549-561.

[12] S.V. Egeland, L. Reubsaet, E. Paus, T.G. Halvorsen, Dual-immuno-MS technique for improved differentiation power in heterodimeric protein biomarker analysis: determination and differentiation of human chorionic gonadotropin variants in serum, Anal. Bioanal. Chem., 408 (2016) 7379-7391. [13] H. Lund, K. Løvsletten, E. Paus, T.G. Halvorsen, L. Reubsaet, Immuno-MS Based Targeted Proteomics: Highly Specific, Sensitive, and Reproducible Human Chorionic Gonadotropin Determination for Clinical Diagnostics and Doping Analysis, Anal. Chem., 84 (2012) 7926-7932. [14] H. Lund, E. Paus, P. Berger, U.-H. Stenman, T. Torcellini, T. Halvorsen, L. Reubsaet, Epitope analysis and detection of human chorionic gonadotropin (hCG) variants by monoclonal antibodies and mass spectrometry, Tumor Biol., 35 (2014) 1013-1022.

[15] H. Lund, A. Snilsberg, E. Paus, T. Halvorsen, P. Hemmersbach, L. Reubsaet, Sports drug testing using immuno-MS: clinical study comprising administration of human chorionic gonadotropin to males, Anal. Bioanal. Chem., 405 (2013) 1569-1576.

[16] H. Lund, A.H. Snilsberg, T.G. Halvorsen, P. Hemmersbach, L. Reubsaet, Comparison of newly developed immuno-MS method with existing DELFIA ${ }^{\circledR}$ immunoassay for human chorionic gonadotropin determination in doping analysis, Bioanalysis, 5 (2013) 623-630.

[17] H. Lund, S.B. Torsetnes, E. Paus, K. Nustad, L. Reubsaet, T.G. Halvorsen, Exploring the Complementary Selectivity of Immunocapture and MS Detection for the Differentiation between hCG Isoforms in Clinically Relevant Samples, J. Proteome Res., 8 (2009) 5241-5252.

[18] C. Rosting, A. Gjelstad, T.G. Halvorsen, Water-Soluble Dried Blood Spot in Protein Analysis: A Proof-of-Concept Study, Anal. Chem., 87 (2015) 7918-7924. 
[19] E. Paus, K. Nustad, Immunoradiometric assay for alpha gamma- and gamma gamma-enolase (neuron-specific enolase), with use of monoclonal antibodies and magnetizable polymer particles, Clinical Chemistry, 35 (1989) 2034-2038.

[20] K.O. Svendsen, H.R. Larsen, S.A. Pedersen, I. Brenna, E. Lundanes, S.R. Wilson, Automatic filtration and filter flush for robust online solid-phase extraction liquid chromatography, Journal of

417 Separation Science, 34 (2011) 3020-3022.

418 [21] A. Lempiäinen, K. Hotakainen, H. Alfthan, U.-H. Stenman, Loss of human chorionic gonadotropin in urine during storage at $-20^{\circ} \mathrm{C}$, Clinica Chimica Acta, 413 (2012) 232-236. Assay to Measure Prenatal Screening Markers Pregnancy-Associated Plasma Protein A and Free $\beta$ - 


\section{Figure captions}

425 Figure 1: MS chromatograms of the hCG $\beta$-T5 peptide after preparation of blank (non-spiked) DMS 426 (lower), and DMS spotted with samples spiked at low levels (20 IU/L (58 pM), upper) using DMPK-C

427 as sampling material

428 Figure 2: MS chromatograms of the hCG $\beta$-T5 peptide after preparation of blank (non-spiked) DMS

429 (lower), and DMS spotted with samples spiked at low levels (20 IU/L (58 pM), upper) using CMC as 430 sampling material.

431 Figure 3: Analysis of serum sample from patient previously diagnosed with testicular cancer. Fifteen 432 microliter of serum was spotted on either DMPK-C (a) and CMC (b). MS/MS spectrum of the hCG $\beta$-T5 433 peptide is included. 

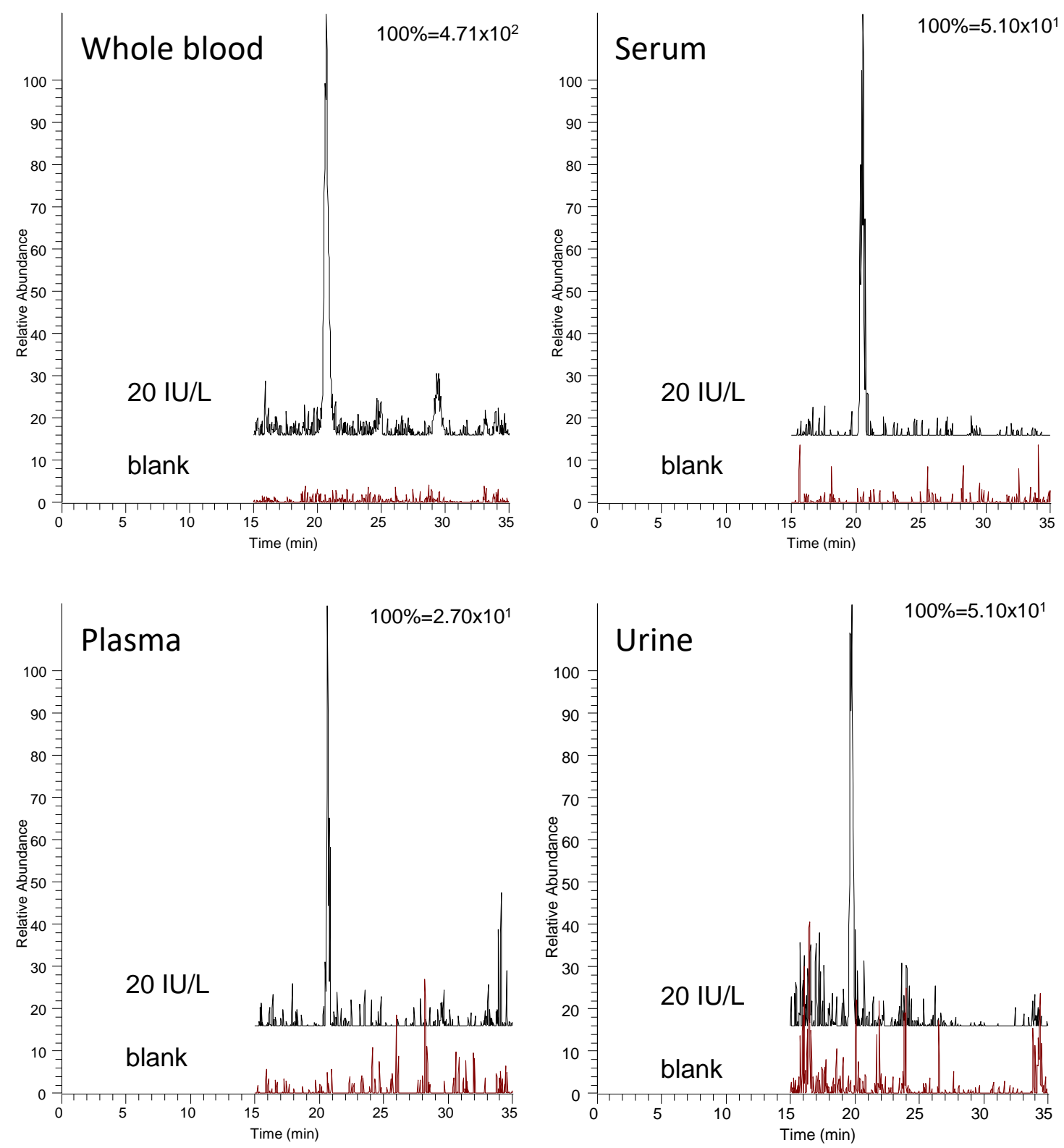

\section{Figure 1}



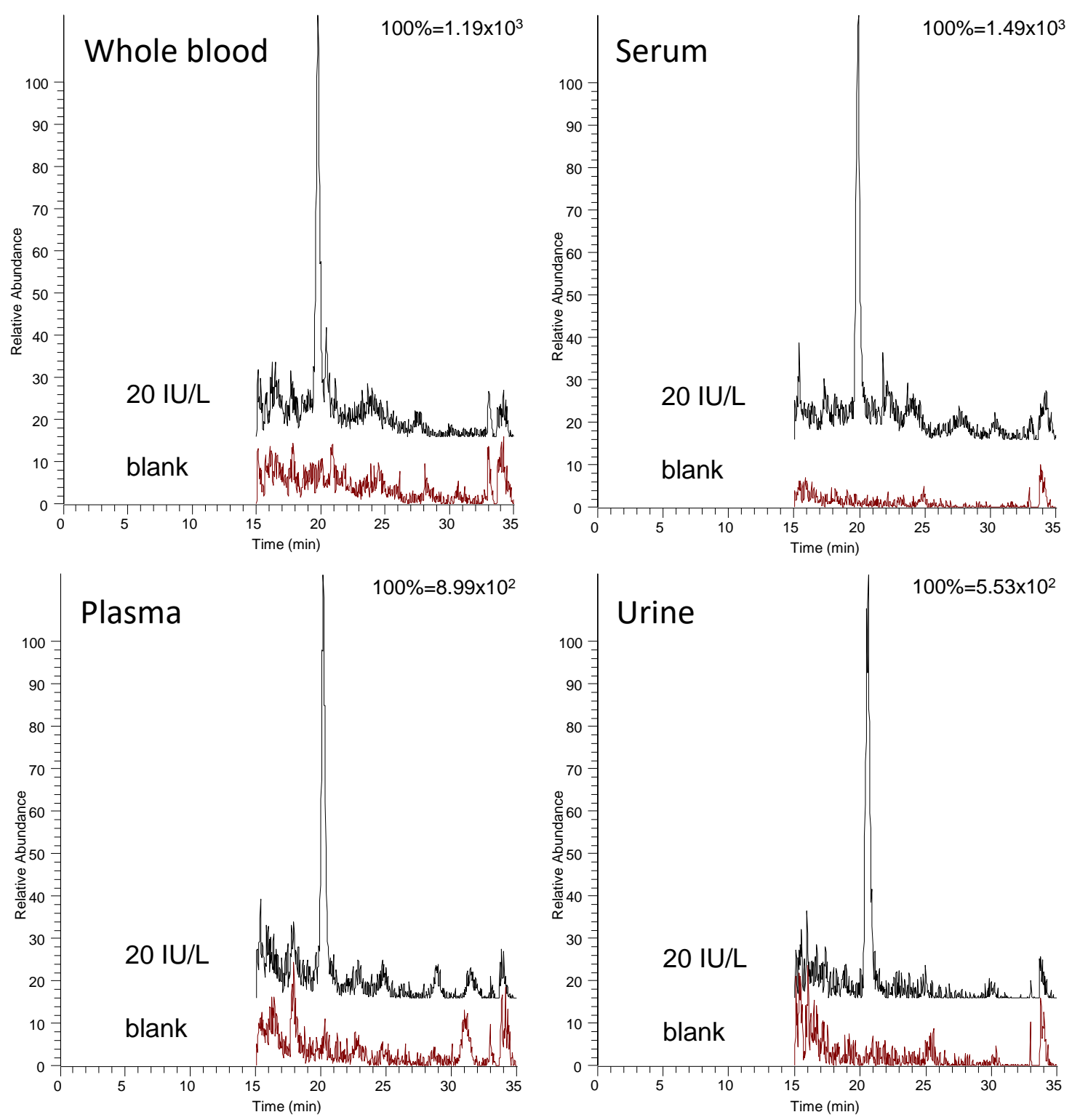

Figure 2 

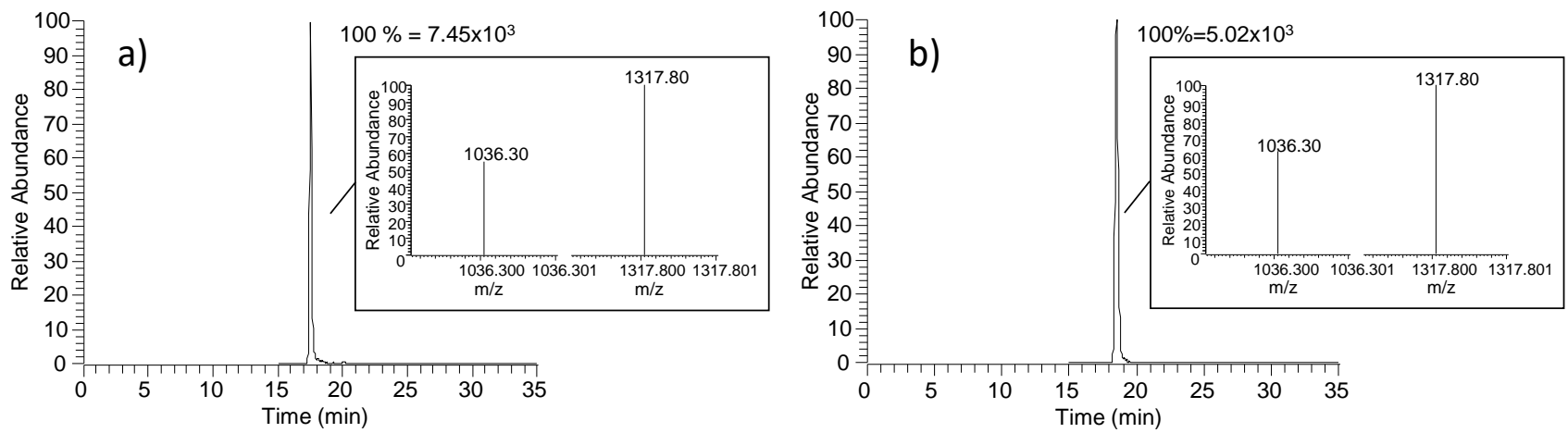

Figure 3 
Table 1. Overview of the detection limits obtained using the different sampling materials and sample matrices

\begin{tabular}{lll}
\hline Sampling material & Sample matrix & Limit of detection $^{1}(\mathrm{IU} / \mathrm{L}(\mathrm{pM}))$ \\
\hline DMPK-C & Whole blood & $5.0(14.5)$ \\
& Plasma & $8.9(25.8)$ \\
& Serum & $7.8(22.6)$ \\
$\mathrm{CMC}$ & Urine & $10.5^{2}(30.5)^{2}$ \\
& Whole blood & $4.9(14.2)$ \\
& Plasma & $6.4^{3}(18.6)^{3}$ \\
& Serum & $3.0(8.7)$ \\
& Urine & $2.1(6.1)$ \\
\hline
\end{tabular}

${ }^{1} n=5$ and based on $S / N=3^{2} n=3$ and based on $S / N=3^{3} n=4$ and based on $S / N=3$ 
Table 2. Method evaluation using serum as matrix

\begin{tabular}{|c|c|c|c|c|c|}
\hline \multirow{2}{*}{$\begin{array}{l}\text { Sampling } \\
\text { material }\end{array}$} & \multirow{2}{*}{$\begin{array}{l}\text { Linearity }{ }^{1} \\
\left(r^{2}\right)\end{array}$} & \multicolumn{2}{|c|}{ Repeatability (RSD (\%), n=5) } & \multicolumn{2}{|c|}{ Accuracy $(\%, n=5)$} \\
\hline & & $\begin{array}{l}\text { Low } \\
\text { concentration }^{2}\end{array}$ & $\begin{array}{l}\text { High } \\
\text { concentration }^{3}\end{array}$ & $\begin{array}{l}\text { Low } \\
\text { concentration }^{2}\end{array}$ & $\begin{array}{l}\text { High } \\
\text { concentration }^{3}\end{array}$ \\
\hline DMPK-C & 0.93 & 13 & 29 & $106 \pm 16$ & $97 \pm 28$ \\
\hline $\mathrm{CMC}$ & 0.94 & 31 & 29 & $96 \pm 25$ & $95 \pm 28$ \\
\hline
\end{tabular}

${ }^{1}$ Range 20-1000 IU/L (58-2900 pM) for DMPK-C and 10-1000 IU/L (29-2900 pM) for CMC, $n=3$.

${ }^{2} 20 \mathrm{IU} / \mathrm{L}$ (58 pM) for DMPK-C and $10 \mathrm{IU} / \mathrm{L}$ (29 pM) for CMC

${ }^{3} 1000 \mathrm{IU} / \mathrm{L}(2900 \mathrm{pM})$ for both DMPK-C and CMC 